\title{
Observation of magnetization waves in negative-index photonic metamaterials
}

\author{
G. Dolling ${ }^{\text {a) }}$ and M. Wegener \\ Institut für Angewandte Physik, Universität Karlsruhe (TH), D-76131 Karlsruhe, Germany \\ and DFG-Center for Functional Nanostructures (CFN), Universität Karlsruhe (TH), \\ D-76131 Karlsruhe, Germany
}

A. Schädle and S. Burger

Zuse Institute Berlin, Takustrasse 7, D-14195 Berlin, Germany

and DFG Forschungszentrum Matheon, Technische Universität Berlin, D-10623 Berlin, Germany

\begin{abstract}
S. Linden
Institut für Nanotechnologie, Forschungszentrum Karlsruhe in der Helmholtz-Gemeinschaft, D-76021 Karlsruhe, Germany
\end{abstract}

(Received 4 October 2006; accepted 26 October 2006; published online 8 December 2006)

\begin{abstract}
By measuring and calculating oblique-incidence transmittance spectra of a negative-index photonic metamaterial layer, the authors infer the in-plane dispersion relation of the magnetization wave. From the geometry and the dispersion shape, the authors conclude that coupling is predominantly via magnetic dipoles for wave propagation along the magnetic-dipole direction. These magnetization waves are the classical analog of magnon excitations of quantum-mechanical spins. (C) 2006 American Institute of Physics. [DOI: 10.1063/1.2403905]
\end{abstract}

In the years 2005 and 2006, metamaterials with a negative index of refraction $n$ have become available in the optical regime. ${ }^{1-4}$ The negative index results from tailoring the properties of nanostructured metallic building blocks, "photonic atoms," the metamaterial is composed of. In particular, electric atoms with an electric-dipole moment leading to a negative electric permittivity ${ }^{5} \epsilon$ and magnetic atoms with a magnetic-dipole moment leading to a negative magnetic permeability $^{6,7} \mu$ have to be combined and densely packed into an effective material. ${ }^{8}$ To a first approximation, the optical properties of the metamaterial are the result of the optical properties of the individual photonic atoms. In other words, interactions among the different unit cells can be neglected.

One step beyond that, the electric dipole-dipole and magnetic dipole-dipole interactions between next-nearest neighbors (and beyond) lead to a coupling of the photonic atoms, thus modifying the material optical properties. The aim of this letter is to measure the resulting dispersion relations and to clarify the nature of the corresponding waves.

In the present context, one can distinguish between four different types of couplings: On the one hand, electric $(e)$ and magnetic $(m)$ dipole-dipole interactions, respectively; on the other hand, transverse $(\perp)$ and longitudinal $(\|)$ waves, respectively. Here, transverse and longitudinal refer to the dipole orientation with respect to the in-plane wave vector $\mathbf{k}_{\|}=\left(k_{x}, k_{y}\right)$ of the excitation. The theory for all four cases has been evaluated. ${ }^{9,10}$

Intuitively, the interaction between equally oriented dipoles-be it electric or magnetic - is attractive (repulsive) for a spatially homogeneous longitudinal (transverse) arrangement. Thus, the energetic minimum (maximum) of the dispersion relation $E\left(\mathbf{k}_{\|}\right)$occurs for zero in-plane momentum $\mathbf{k}_{\|}$, and finite wave numbers increase (decrease) the energy. Note that electric and magnetic dipoles are usually perpen-

${ }^{a)}$ Electronic mail: gunnar.dolling@physik.uni-karlsruhe.de

dicular to each other. This means that, e.g., the electric transverse case competes with the magnetic longitudinal case. Thus, the photon energy increases or decreases with wave number, depending on whether electric or magnetic dipoledipole interactions dominate. The question which one dominates cannot easily be answered a priori. Investigating this question is interesting in itself and also a sensitive test of our current understanding of negative-index photonic metamaterials.

Here, we study the negative-index metamaterial design that has led to the best performance in the optical regime so far. It is shown in Fig. 1. The sample investigated here is closely similar but not identical to that used in Ref. 4. The sample parameters are given in the caption of Fig. 1. Coupling to the above mentioned modes is accomplished by oblique incidence of light onto the metamaterial layer. This geometry leads to a modulus of the in-plane wave number $k_{\|}$ given by

$$
k_{\|}=\frac{2 \pi}{\lambda} \sin (\alpha),
$$

with the incident (vacuum) wavelength of light $\lambda$. By choosing different axes of rotation, different principal directions in reciprocal space can be investigated. The insets in Fig. 2 illustrate the two cases $\mathbf{k}_{\|}=k_{\|}(1,0)$ and $k_{\|}(0,1)$. For the sample parameters given in Fig. 1(b), for $\alpha=40^{\circ}$ angle of incidence with respect to the surface normal and for $\lambda$ $=1500 \mathrm{~nm}$, the modulus of the in-plane wave vector reaches a value of greater than $k_{\|}=0.5 \pi / a$. This means that a substantial fraction of the first Brillouin zone can be investigated along these lines.

Experimental transmittance spectra are shown in Fig. 2(a) for the polarization and geometry indicated on the top. The transmittance dips directly arise from the magneticdipole resonance, and the featureless electric response is that of a diluted Drude metal with $\operatorname{Re}(\epsilon(\omega))<0$ for the entire spectral region shown (also see Ref. 8). For both obliqueEVA-STAR (Elektronisches Volltextarchiv - Scientific Articles Repository) http://digbib.ubka.uni-karlsruhe.de/volltexte/1000010974 
(a)
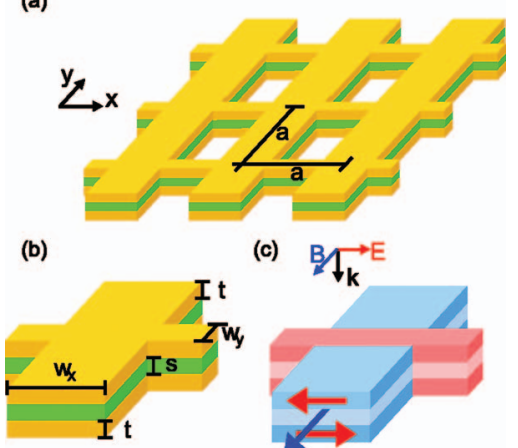

(c) $\vec{P} \overrightarrow{\imath_{\mathrm{k}}}$

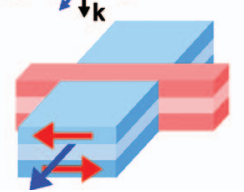

FIG. 1. (Color) (a) Scheme of negative-index metamaterial investigated. (b) Magnified view of one unit cell. Constituent materials are gold (yellow) and $\mathrm{MgF}_{2}$ (green). Parameters are $a=605 \mathrm{~nm}, w_{x}=324 \mathrm{~nm}, w_{y}=100 \mathrm{~nm}$, $t=30 \mathrm{~nm}$, and $s=32 \mathrm{~nm}$. (c) The "magnetic atom" (blue) and the "electric atom" (red) in one unit cell are highlighted. The blue and red arrows schematically indicate a snapshot of the magnetic- and electric-dipole vectors, respectively.

incidence geometries shown in Fig. 2, the transmittance dip shifts towards higher photon energies with increasing angle $\alpha$.

To further analyze these findings, we plot the photonenergy position of the transmittance dips (see vertical dashed lines in Fig. 2) versus in-plane wave number $k_{\|}$. The latter can easily be calculated from the angle $\alpha$ via Eq. (1). The resulting measured dispersion relations are shown in Fig. 3. It has been thoroughly discussed for the structure under investigation that the electric dipoles are oriented along the $x$ direction and that the magnetic-dipole moments are oriented along the $y$ direction ${ }^{4}$ [also see Fig. 1(c)]. Thus, the spectral shape of the dispersion relations indicates that longitudinal electric dipole-dipole coupling is dominant for propagation along $k_{\|}(1,0)$, whereas longitudinal magnetic dipole-dipole coupling is dominant for propagation along $k_{\|}(0,1)$ (otherwise the photon energy in Fig. 3 should decrease rather than increase with increasing modulus of the in-plane wave number). Waves resulting from magnetic dipole-dipole interactions have recently been discussed theoretically and have been called "magnetoinductive" waves. ${ }^{9}$ These magnetiza-

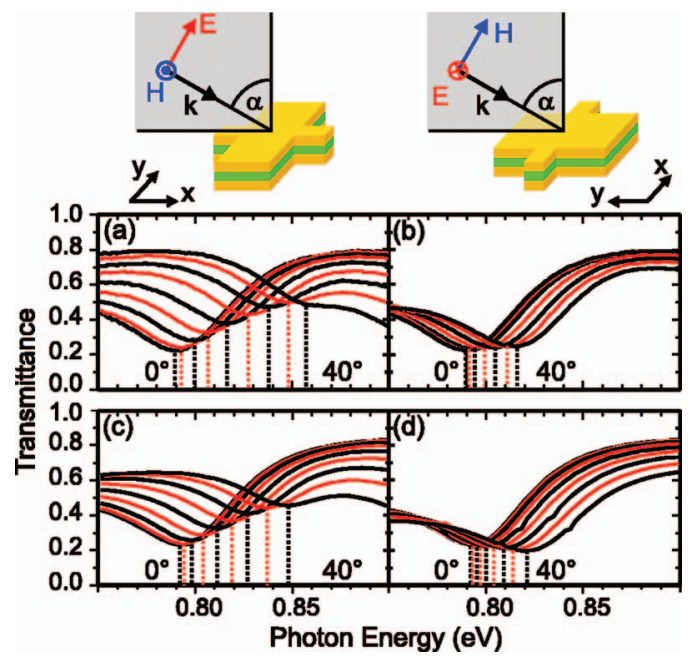

FIG. 2. (Color) [(a) and (b)] Measured oblique-incidence transmittance spectra for different angles $\alpha$ with respect to the surface normal, from $0^{\circ}$ to $40^{\circ}$ in steps of $5^{\circ}$ (for clarity, the color alternates between black and red). The geometry is indicated on the top. [(c) and (d)] Calculated spectra cor-

responding to (a) and (b), respectively.
Downloaded 19 Mar 2009 to 129.13 .72 .198$. Redistribution subject to AlP license or copyright; see http://apl.aip.org/apl/copyright.jsp
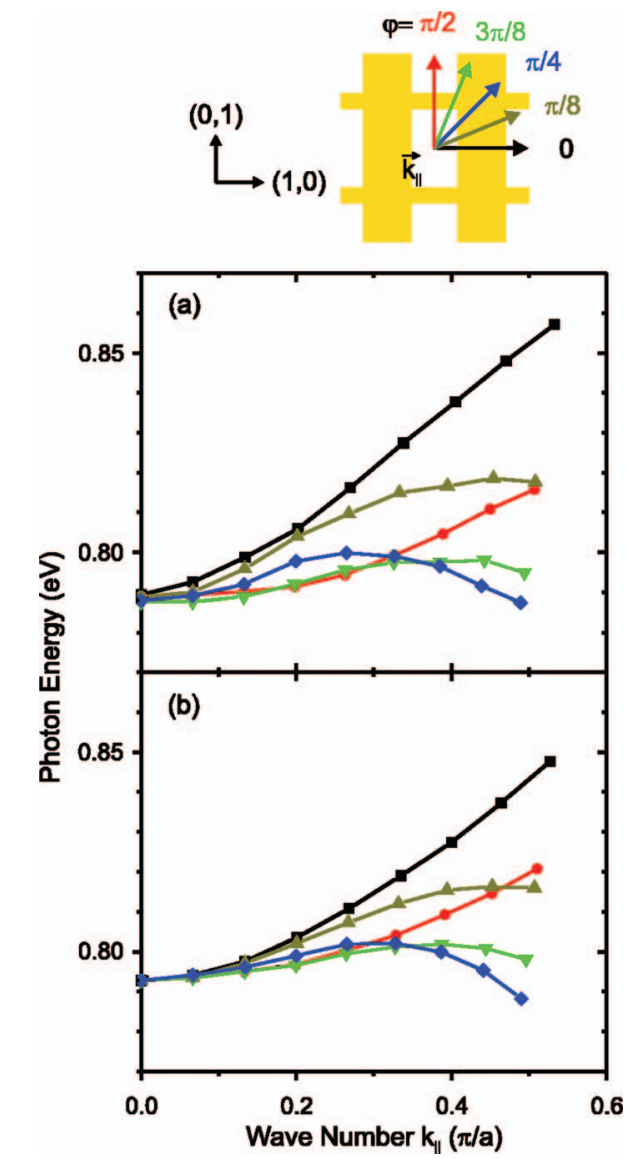

FIG. 3. (Color) Photon energies of transmittance dips (see vertical dashed (a) Experiment, (b) theory. Propagation directions are indicated by the inplane wave vector $\mathbf{k}_{\|}=k_{\|}(\cos (\varphi), \sin (\varphi))$ and illustrated at the top. $\varphi=0$ corresponds to Figs. 2(a) and 2(c) and $\varphi=\pi / 2$ to Figs. 2(b) and 2(d).

tion waves are the classical analog of magnons for interacting quantum-mechanical spins. So far, these waves have only been observed experimentally at megahertz frequencies. ${ }^{11}$ Figure 3 also shows propagation directions other than the two principal directions $\mathbf{k}_{\|}=k_{\|}(1,0)$ and $\mathbf{k}_{\|}=k_{\|}(0,1)$. Here, the behavior is more complex and the photon energy versus in-plane momentum is a nonmonotonous function. We interpret this behavior in terms of the nontrivial competition between the four types of interactions (see discussion above): electric/magnetic and transverse/longitudinal.

The spectral behavior at larger photon energies (not shown) is rather complex and dominated by various Rayleigh anomalies, which are not at all related to the magneticdipole resonance. To verify that all spectral features discussed in Figs. 2 and 3 are actually directly related to the magnetic-dipole resonance, we have fabricated another set of samples for which the upper of the two gold layers (see Fig. 1) is absent. Indeed, the transmittance dips shown in Fig. 2 completely disappear and near-unity transmittance is obtained for all photon energies and angles $\alpha$ in the range of Fig. 2. In contrast, the various Rayleigh anomalies at higher photon energies hardly change at all.

To further test our interpretation, we compare the measured data with numerical calculations based on an advanced frequency-domain finite-element code. Blochperiodic boundary conditions are applied in the $x$ and $y$ directions $^{12}$ on a square lattice with lattice constant $a$ to allow for oblique incidence of light. In the $\pm z$ direction we lines in Fig. 2) vs in-plane wave number $k_{\|}$as determined from the angle $\alpha$. 
apply transparent boundary conditions based on the perfectly matched layer method. ${ }^{13,14} \mathrm{We}$ discretize a unit cell with an unstructured mesh of about 600 prisms. Maxwell's equations are discretized using vectorial finite elements (Whitney elements) of second order, leading to a sparse matrix equation with about 130000 unknowns.

Geometrical parameters are as in Fig. 1. For the permittivity of gold we assume a frequency dependence according to the Drude model, with a plasma frequency of $\omega_{\mathrm{pl}}=1.33$ $\times 10^{16} \mathrm{~s}^{-1}$ and a collision frequency of $\omega_{\mathrm{c}}=1.15 \times 10^{14} \mathrm{~s}^{-1}$. The relative permittivity of the glass substrate is taken as 2.25 and that of the $\mathrm{MgF}_{2}$ spacer layer as 1.90 .

Calculated results are shown in Figs. 2 and 3. Obviously, very good agreement between experiment and theory is obtained. In particular, the calculated dispersion relations quantitatively agree with the experimental ones and reproduce the observed anisotropy regarding propagation along the two principal directions $\mathbf{k}_{\|}=k_{\|}(1,0)$ and $\mathbf{k}_{\|}=k_{\|}(0,1)$ as well as for the other three directions.

Finally, we address the question which parts of the negative-index structure are important for the observed couplings. If, for example, the thin metal double wires [marked red in Fig. 1(c)] are completely eliminated in the calculations, the transmittance dips in Fig. 2 hardly show any influence on $\alpha$ (not shown). This observation indicates that the thin metal double wires mediate both the above-mentioned strong electric and magnetic dipole-dipole couplings. This observation also unambiguously shows that the observed spectral shifts are not a property of the isolated magnetic atoms.

In conclusion, we have investigated the dispersion relation of in-plane waves in negative-index photonic metamaterials arising from the coupling of the photonic atoms the metamaterial is composed of. The longitudinal magnetic waves are the classical analog of quantum-mechanical spin waves, well known as magnon excitations. Regarding experi- ments in the optical regime, such magnetization waves are observed here for the first time to the best of our knowledge. For other propagation directions, we find a complex interplay between magnetization and polarization waves.

The authors acknowledge support by the Deutsche Forschungsgemeinschaft (DFG) and the State of BadenWürttemberg through the DFG-Center for Functional Nanostructures (CFN) within subproject A1.5. The authors acknowledge support through the DFG-Center Mathematics for Key Technologies (Matheon). The research of one of the authors (S.L.) is further supported through a "HelmholtzHochschul-Nachwuchsgruppe" (VH-NG-232).

${ }^{1}$ S. Zhang, W. Fan, N. C. Panoiu, K. J. Malloy, R. M. Osgood, and S. R. J. Brueck, Phys. Rev. Lett. 95, 137404 (2005).

${ }^{2}$ V. M. Shalaev, W. Cai, U. K. Chettiar, H. Yuan, A. K. Sarychev, V. P. Drachev, and A. V. Kildishev, Opt. Lett. 30, 3356 (2005).

${ }^{3}$ G. Dolling, C. Enkrich, M. Wegener, C. M. Soukoulis, and S. Linden, Science 312, 892 (2006).

${ }^{4}$ G. Dolling, C. Enkrich, M. Wegener, C. M. Soukoulis, and S. Linden, Opt. Lett. 31, 1800 (2006).

${ }^{5}$ J. B. Pendry, A. J. Holden, W. J. Stewart, and I. Youngs, Phys. Rev. Lett. 76, 4773 (1996).

${ }^{6}$ J. B. Pendry, A. J. Holden, D. J. Robbins, and W. J. Stewart, IEEE Trans. Microwave Theory Tech. 47, 2075 (1999).

${ }^{7}$ V. A. Podolskiy, A. K. Sarychev, E. E. Narimanov, and V. M. Shalaev, J. Opt. A, Pure Appl. Opt. 7, 32 (2005).

${ }^{8}$ S. Zhang, W. Fan, K. J. Malloy, S. R. J. Brueck, N. C. Panoiu, and R. M. Osgood, Opt. Express 13, 4922 (2005).

${ }^{9}$ E. Shamonina and L. Solymar, J. Magn. Magn. Mater. 300, 38 (2006).

${ }^{10}$ M. L. Brongersma, J. W. Hartman, and H. A. Atwater, Phys. Rev. B 62, R16356 (2000).

${ }^{11}$ M. C. K. Wiltshire, E. Shamonina, I. R. Young, and L. Solymar, Electron. Lett. 39, 215 (2003).

${ }^{12}$ S. Burger, R. Klose, A. Schädle, F. Schmidt, and L. Zschiedrich, Proc. SPIE 5728, 164 (2005).

${ }^{13}$ L. Zschiedrich, R. Klose, A. Schädle, and F. Schmidt, J. Comput. Appl. Math. 188, 12 (2006).

${ }^{14}$ L. Zschiedrich, S. Burger, B. Kettner, and F. Schmidt, Proc. SPIE 6115 , 611515 (2006). 